\title{
An audit of interval cholecystectomy for acute cholecystitis in a low resource healthcare system
}

\author{
GM Makatini, ${ }^{1}$ S Mewa Kinoo, ${ }^{2,3}$ (D) B Singh, ${ }^{3}$ \\ ${ }^{1}$ Edendale Hospital, South Africa \\ ${ }^{2}$ King Edward VIII Hospital, South Africa \\ ${ }^{3}$ Department of Surgery, Nelson R Mandela School of Medicine University of KwaZulu-Natal, South Africa
}

Corresponding author, email: smewakinoo@gmail.com

Background: Early laparoscopic cholecystectomy (ELC) is advocated over open cholecystectomy for acute cholecystitis (AC) as it decreases hospital costs with no increase in morbidity and mortality. The applicability of ELC for AC in a resource limited setting has not been reported. This study reviewed patients with AC at a regional state hospital in South Africa to analyse presentation delays and the related surgical strategies.

Methods: Adult patients admitted to King Edward VIII Hospital with a diagnosis of AC were included in the study. The admission, operation and discharge records were used to collect data. The severity of the cholecystitis, the time taken to present to the referral facility, the time taken to present to the regional hospital, time to operation, type of operation, conversion rates and complications were evaluated. Early presentation was recorded for two different cut-off times within 72 hours or 7 days of symptom onset.

Results: One hundred and thirty-nine patients met the inclusion criteria. The mean age was 47 (range 19-74) years and $86 \%$ were female. Forty-nine per cent of patients presented within 7 days and of these only $4.4 \%$ presented within 72 hours. A median of 64 days from time of admission to operative intervention was noted. All patients were considered for laparoscopic cholecystectomy (LC); there was a conversion rate of $26 \%$. No major complications were documented. Conclusion: Interval laparoscopic cholecystectomy appears a reliable option in under-resourced healthcare systems.

Keywords: acute cholecystitis, early cholecystectomy, interval cholecystectomy, delayed cholecystectomy, underresourced healthcare systems

\section{Introduction}

The management of acute cholecystitis (AC) currently is guided by the Tokyo consensus guidelines 2018. Early laparoscopic cholecystectomy (ELC) (within 72 hours of onset of symptoms) is regarded as the standard of care for patients presenting with $\mathrm{AC}$ since it has similar outcomes when compared to delayed or interval laparoscopic cholecystectomies (ILC). However, this 'standard of care' follows the results from high-income countries where there is early presentation to institutions that are equipped to make a diagnosis and manage the patients.

Despite South Africa being an upper-middle-income country access to healthcare services is dichotomous, with $20 \%$ privately insured patients having access to wellresourced healthcare facilities and $80 \%$ whose access is restricted to under-resourced state-funded facilities. Early presentation of patients with AC to hospitals is difficult due to the financial constraints of patients, lack of clinical infrastructure and poor access to appropriate health services. Furthermore, only a few regional and central hospitals in SA offer an unrestricted laparoscopic cholecystectomy (LC) service. Often patients must first be attended to at local clinics, community health centres and local district hospitals where a lack of ultrasound facility further delays appropriate referral to the larger centres where these operations can be offered.

This study reports the incidence of delayed presentation of $\mathrm{AC}$, its effect on the type of surgery performed and outcomes.

\section{Methods}

A chart review was conducted over a 12-month period between January and December 2013 at King Edward VIII Hospital (KEH), Durban, KwaZulu-Natal (KZN). All adult patients aged 18 years and older admitted with a diagnosis of AC were included. Diagnosis was suspected clinically and was confirmed by sonographic evidence of AC (thickened gallbladder wall, presence of pericholecystic fluid or an ultrasound positive murphy's sign). Inflammatory markers, infective markers and a systemic inflammatory response syndrome (SIRS) response were used to determine severity. The severity of AC was graded according to the Tokyo consensus guidelines 2013: severe grade $\mathrm{AC}$ characterised by organ failure; moderate grade AC characterised by the presence of a $\mathrm{WCC}>18$ 000 , a palpable tender right upper quadrant mass, duration of symptoms $>72$ hours or marked local inflammation (gangrenous or emphysematous); and mild grade $\mathrm{AC}$ 
characterised by the absence of organ failure and presence only of mild inflammation.

The admission notes, operative details and discharge notes of all patients with confirmed diagnosis of AC were reviewed to document the patients' demographic details, the time of onset of symptoms, the severity of cholecystitis and the date of first presentation to a referral facility. Early presentation was recorded for two different cut-off times within 72 hours or 7 days of symptom onset. The date of presentation to $\mathrm{KEH}$, distance from referral centre to $\mathrm{KEH}$, reasons for delayed presentation, proposed operation type (laparoscopic or open), time from presentation at $\mathrm{KEH}$ to operation, number of readmissions prior to surgery, time duration of operation in minutes, conversion from laparoscopic to open procedure, and major and minor complications were also documented.

All patients with missing data were excluded from the study. All data were entered into an Excel spreadsheet. All data were presented in the form of numbers and percentages.

\section{Results}

One hundred and seventy-nine patients' charts with AC were reviewed. Forty patients were excluded due to lack of more than 4 of the key variables being recorded in the notes. One hundred and thirty-nine patients were studied. One hundred

Table 1: Demographic referral and clinical data of acute cholecystitis patients

\begin{tabular}{|c|c|c|}
\hline Variable & Number & Per cent \\
\hline \multicolumn{3}{|l|}{ Age } \\
\hline$<20$ years & 1 & 0.7 \\
\hline $20-40$ & 46 & 33 \\
\hline $41-60$ & 63 & 45 \\
\hline$>60$ years & 29 & 21 \\
\hline \multicolumn{3}{|l|}{ Gender } \\
\hline Male & 19 & 14 \\
\hline Female & 120 & 86 \\
\hline \multicolumn{3}{|c|}{ Referring institute } \\
\hline Self & 32 & 23 \\
\hline Clinic & 61 & 44 \\
\hline District hospital & 46 & 33 \\
\hline \multicolumn{3}{|c|}{ Duration of symptoms } \\
\hline $0-3$ days & 20 & 14 \\
\hline 3-7 days & 49 & 35 \\
\hline$>7$ days & 70 & 50 \\
\hline \multicolumn{3}{|c|}{ Distance from KEH } \\
\hline$<5 \mathrm{~km}$ & 16 & 12 \\
\hline $5-10 \mathrm{~km}$ & 60 & 43 \\
\hline $11-15 \mathrm{~km}$ & 12 & 9 \\
\hline $16-20 \mathrm{~km}$ & 30 & 22 \\
\hline$>20 \mathrm{~km}$ & 21 & 15 \\
\hline \multicolumn{3}{|c|}{ Admissions before operation } \\
\hline 1 & 112 & 81 \\
\hline 2 & 24 & 17 \\
\hline 3 & 3 & 2 \\
\hline$>3$ & 0 & 0 \\
\hline
\end{tabular}

KEH - King Edward the VIII Hospital and twenty patients were female $(86 \%)$, and 19 patients were male $(14 \%)$. The mean age was 47 years with a range from 19 to 74 years. The average symptom duration (from initial onset) before presentation to a nearest healthcare facility was 16.6 days; the average symptom duration (onset of symptoms) before presentation to $\mathrm{KEH}$ was 20 days. The average distance from referral centres, where patients initially presented, to $\mathrm{KEH}$, was $31.6 \mathrm{~km}$ with a range from $1.8 \mathrm{~km}$ to $197 \mathrm{~km}$. $86 \%$ of patients qualified as late presentation using the 72 hour cut-off and $51 \%$ when using the 7 day cut-off. Most reasons for late presentations were not documented. Transport challenges from rural areas; no guardians for children that will be left unattended at home; inability to afford travel and delay in diagnosis at referral centres were amongst the documented reasons for late presentations. Ninety-four per cent of patients presented with mild cholecystitis and the remaining $6 \%$ with moderate cholecystitis. No patients presented with severe cholecystitis. The majority of patients had one admission before operation $(81 \%)$. The readmission rate whilst awaiting delayed cholecystectomy (DLC) was 19\%. There were 21 patients who had a total of two admissions and three patients had three admissions, one of whom was a defaulter (Table 1).

All but one patient underwent interval cholecystectomy including patients presenting within 72 hours to a week due to the lack of an emergency theatre facility at KEH. The average number of days from admission to operation was 70 days. The mean was 62 days (IQR 39-96) with a minimum of 24 days and a maximum of 243 days. Of the 139 patients, 117 patients were scheduled for LC; the remaining 22 for open cholecystectomies (OC) because of previous open abdominal surgery and not because of

\begin{tabular}{|c|c|c|}
\hline Variable & Number & Per cent \\
\hline \multicolumn{3}{|l|}{ Timing of surgery } \\
\hline Early & 1 & 0.72 \\
\hline Interval & 138 & 99 \\
\hline \multicolumn{3}{|l|}{ Disease severity } \\
\hline Mild & 131 & 94 \\
\hline Moderate & 8 & 6 \\
\hline Severe & 0 & 0 \\
\hline \multicolumn{3}{|l|}{ Initial surgical option } \\
\hline Laparoscopic & 117 & 84 \\
\hline Open & 22 & 16 \\
\hline Conversions to open & 30 & 26 \\
\hline \multicolumn{3}{|c|}{ Length of stay post cholecystectomy } \\
\hline $1-3$ & 114 & 82 \\
\hline $4-7$ & 21 & 15 \\
\hline$>7$ & 4 & 3 \\
\hline \multicolumn{3}{|l|}{ Operative time } \\
\hline \multicolumn{3}{|c|}{ Started and completed laparoscopically } \\
\hline \multicolumn{3}{|c|}{$\begin{array}{l}\text { Started laparoscopically completed } \\
\text { open }\end{array}$} \\
\hline Laparoscopic overall & $\begin{array}{c}80.2 \pm \\
30.5 \\
\text { minutes }\end{array}$ & \\
\hline Open & $\begin{array}{l}80 \pm 31 \\
\text { minutes }\end{array}$ & \\
\hline
\end{tabular}


the timing of presentation. Of the 117 LCs undertaken, 87 (74\%) were completed laparoscopically and 30 (25\%) were converted to OC. The reasons for conversion included dense adhesions encountered at surgery, unclear anatomy, the inability to achieve Strasberg's critical view of safety, gallbladder perforation with gross stone spillage, empyema and excessive bleeding from the liver bed.

The average time for LC was 80.2 minutes (range 25180 minutes). The average time taken for the $52 \mathrm{OC}$ was 80 minutes (range 30-150 minutes). The average hospital stay time post interval cholecystectomy was 48 hours. There were no major procedure-related complications documented (Table 2).

\section{Discussion}

The gold standard of management of AC is a laparoscopic cholecystectomy. ${ }^{1}$ The timing of this operation depends largely on the time of presentation, the severity of cholecystitis, institutional capability and general patient condition as noted in the Tokyo guidelines.

Cholecystectomy may be undertaken early following symptom onset and presentation or can be delayed and performed as an interval cholecystectomy, i.e. after the acute episode has settled and the patient discharged to return for an elective LC.

In the current literature there is no standard definition of what constitutes an early cholecystectomy. Early cholecystectomy has been variably referred to as a cholecystectomy undertaken anywhere within 24 hours to within 10 days of onset of symptoms, but not after 10 days. ${ }^{2}$ The most widely quoted time intervals within this range are 24 hours, 72 hours and 7 days from onset of symptoms.

Definitions for delayed or interval cholecystectomy generally refer to intervention anytime between 10 days and 45 days, but may be as long as 12 weeks after initial symptom onset. ${ }^{2}$ One report recommends that operation between 10 days and 45 days should be avoided, as the adhesions from inflammation are dense and vascular, resulting in higher morbidity and prolonged hospital stays (5 days longer) when compared to both early cholecystectomies (less than 10 days) and interval cholecystectomies (more than 45 days). ${ }^{3}$ The conversion rates, however, are comparable. The debate has centred rather around the choice of early cholecystectomy (within 10 days of onset of symptoms) as opposed to delayed cholecystectomy (after 45 days of initial symptom onset).

ELC was initially contraindicated in acute cholecystitis, in contrast to patients with uncomplicated gallstone disease without cholecystitis. ${ }^{4}$ This approach was justifiable in the light of some studies revealing early cholecystectomy to be more technically challenging during the acute inflammatory process with longer operative times and a trend towards more bile duct injuries, the most feared complication in laparoscopic cholecystectomy. ${ }^{5}$ However, this has not been universally accepted and several studies have refuted these assertions. ${ }^{6}$

The 2013 Tokyo consensus guidelines and other well recognised studies advocated that ELC be the standard of care for patients presenting early with acute cholecystitis without severe local inflammation, in centres with surgical expertise. A meta-analysis showed similar complication and conversion rates when comparing early versus interval cholecystectomies, with a shorter hospital stay by 4 days in the early group ${ }^{7}$ another trial (not included in this meta-analysis) showed similar results. ${ }^{8}$ Of these early cholecystectomies (performed within 10 days), one study showed that the earlier the operation, the shorter the hospital stay. ${ }^{9}$ A large database including 95000 patients demonstrated that the earlier the operation within this time period (10 days), the fewer the complications. ${ }^{10}$ Similar results were revealed in a population based analysis involving 4113 patients with AC which showed the benefits of doing cholecystectomy within the first 48 hours of onset of symptoms. ${ }^{11}$

The early procedure offers the convenience of resolving the patient's pathology during a single admission. This approach has proven beneficial in reducing hospital costs, low conversion rates, reducing length of time away from work and does not increase the risk of complications compared to delayed intervention.

Despite evidence pointing to a successful outcome with early cholecystectomy, a recent study from Durban, KwaZulu-Natal, concluded that most patients $(88.2 \%)$ had presented late ( $>72$ hours) and thus DLC was their standard practice. ${ }^{12}$ The findings in our study reveal very similar results with $85.61 \%$ of patients presenting late ( $>72$ hours), all of whom underwent delayed cholecystectomies. With a postoperative average hospital stay of 48 hours and no major procedure related complications, a conversion rate of $25.6 \%$ and an average laparoscopic cholecystectomy time of 80.2 minutes, these data are comparable to those quoted for early cholecystectomy.

A limitation of our paper is its retrospective design nature and poor documentation of data (reflective in the large number of excluded patients). Even in the non-excluded patients, minor non-procedure related complications were not recorded. We regard the lack of major procedure related complications as attributable to our high conversion rate to obviate the technical challenges posed by advanced inflammation, dense vascular adhesions, and distorted anatomy, but appreciate that this may also reflect poor documentation and that ongoing audits of our cholecystectomy practice must prospectively document all complications in a systematic manner.

In our study there was a re-admission rate of $19.4 \%$. Although re-admissions add to overall hospital stay, a study comparing same admission versus DLC for patients presenting with $\mathrm{AC}$ after 7 days concluded that overall delay can be reduced as DLC resulted in lower conversion rate and shorter hospital stay for this category of patients. ${ }^{13}$

In many developing countries complex technology is not readily available and laparoscopic services are extremely limited. ${ }^{14}$ Despite our privileged position in sub-Saharan Africa this still pertains in $\mathrm{KEH}$, a major South African regional hospital. At $\mathrm{KEH}$ there is a single emergency operating room run by two emergency nursing teams and a single anaesthetist. This single emergency operating room covers all general surgery, trauma, gynaecological, ENT, urological and orthopaedic emergencies. Consequently, surgical emergencies take precedence over patients presenting with mild to moderate cholecystitis. For these reasons the implementation of ELC in our setting has many challenges. However, the results from this audit show that a DLC approach appears safe in resource constrained environments and merits further prospective analysis. 


\section{Conflict of interest}

The authors have no conflict of interest to declare.

\section{Funding source}

None.

\section{Ethics approval}

Full ethical approval was obtained from the Biomedical Research Ethics Committee (BREC) of the University of KwaZulu-Natal (ref no. BE347/14) and permission to conduct research obtained from the KZN Department of Health's Research Unit and the KEH Management.

\section{ORCID}

S Mewa Kinoo (D) https://orcid.org/0000-0002-4596-022X B Singh (iD https://orcid.org/0000-0003-0799-256X

\section{REFERENCES}

1. Glavic Z, Begic L, Simlesa D, Rukavina A. Treatment of acute cholecystitis. A comparison of open vs laparoscopic cholecystectomy. Surg Endosc. 2001;15:398-401.

2. Ansaloni L, Pisano M, Coccolini F, et al. 2016 WSES guidelines on acute calculous cholecystitis. World J Emerg Surg. 2016;11:25.

3. Gutt CN, Encke J, Koninger J, et al. Acute cholecystitis: early versus delayed cholecystectomy, a multicenter randomized trial (ACDC study, NCT00447304). Ann Surg. 2013;258(3):385-93.

4. Macafee DAL, Humes DJ, Bouliotis G, et al. Prospective randomized trial using cost-utility analysis of early versus delayed laparoscopic cholecystectomy for acute gallbladder disease. Br J Surg. 2009;96(9):1031-40.

5. Soderlund C, Frozanpor F, Linder S. Bile duct injuries at laparoscopic cholecystectomy: a single-institution prospective study. Acute cholecystitis indicates an increased risk. World J Surg. 2005;29:987-93.

6. Johansson M, Thune A, Blomqvist A, et al. Management of Acute Cholecystitis in the Laparoscopic Era: Results of a Prospective, Randomized Clinical Trial. J Gastrointest Surg. 2003;7:642-5

7. Gurusamy KS, Davidson C, Gluud C, Davidson BR. Early versus delayed laparoscopic cholecystectomy for people with acute cholecystitis. Cochrane Database Syst Rev. 2013;6. https://doi.org/10.1002/14651858. CD005440.pub3.

8. Mare LD, Saadi A, Roulin D, Demartines N, Halkic N. Delayed versus early laparoscopic cholecystectomy for acute cholecystitis: A prospective randomized study. HPB. 2012;14:130.

9. Chandler CF, Lane JS, Ferguson P, Thompson JE, Ashley SW Prospective evaluation of early versus delayed laparoscopic cholecystectomy for treatment of acute cholecystitis. Am Surg. 2000;66(9):896-900.

10. Zafar SN, Obirize A, Adesibikan B, et al. Optimal Time for Early Laparoscopic Cholecystectomy for Acute Cholecystitis. JAMA. 2015;150(2):129-36.

11. Banz V, Gsponer T, Candinas D, Güller U. Population-based analysis of 4113 patients with acute cholecystitis: defining the optimal time-point for laparoscopic cholecystectomy. Ann Surg. 2011 Dec;254(6):964-70.

12. Mbatha SZ, Anderson F. Outcomes in laparoscopic cholecystectomy in a resource constrained environment. S Afr J Surg. 2016;54(3).

13. Tan JKH, Goh JCI, Lim JWL, et al. Delayed Presentation of Acute Cholecystitis: Comparative Outcomes of SameAdmission Versus Delayed Laparoscopic Cholecystectomy. J Gastroint Surg. 2017;21(5):840-5.

14. Afuwape OO, Akute OO. The challenges and solutions of laparoscopic surgical practice in the developing countries. Niger Postgrad Med J. 2011;18:197-19. 